EESTI NSV TEADUSTE AKADEEMIA TOIMETISED.

FOUSIKA. MATEMAATIKA

ИЗВЕСТИЯ АКАДЕМИИ НАУК ЭСТОНСКОП ССР, ФИЗИКА. МАТЕМАТИКА

PROCEEDINGS OF THE ACADEMY OF SCIENCES OF THE ESTONIAN SSR.

PHYSICS. MATHEMATICS

$1984,33,2$

R.-K. LOIDE, P. SUURVARIKK

удК 539.12

\title{
ON THE SPINOR SUPERFIELD EQUATIONS OF MOTION
}

\author{
(Presented by $H$. Keres)
}

The structure of spinor superfield equations of motion in terms of component fields is analysed. The equations for component fields are reduced to the equations previously investigated in the theory of invariant wave equations. In the case of superspins 1 and 0 , the Dirac equation, multi-mass equations for vector-bispinor and Hurley-type equations are obtained. In the superspin $1 / 2$ case we have the Dirac and Kemmer-Duffin spin 0 and spin 1 equations.

\section{Introduction}

In the theory of superfields, the equations of motion are playing the same role as invariant wave equations in the ordinary field theory. From the existing superfield equations of motion, it becomes clear that they are quite complicated and for that reason have not yet been investigated enough. Also, there are no general methods to derive superfield equations for an arbitrary superspin Y. The root method known in the theory of ordinary wave equations is generalized into the superfield case $\left.{ }^{1}\right]$, and it allows to derive superfield equations for spinor superfield $\left[{ }^{2}\right]$. There are other types of superfield equations, too, as, for instance, the equation for chiral superfield proposed in $\left[^{3}\right]$. For that reason the investigation of different superfield equations of motion is quite important.

In this paper we deal with spinor superfield equations of motion. These equations are given in $\left[{ }^{1-3}\right]$, here we give the analysis of field equations for component fields. We proceed directly from the superfield equations of motion and write down the equations for component fields. Our approach differs from that of paper [ $\left.{ }^{1}\right]$ where the redefinition of field variables has been used. We do not consider this method to be unique for it seems somewhat artificial. It seems that the equations derived directly from superfield equations are quite interesting and can be reduced to the equations previously investigated in the theory of invariant wave equations. So we obtain the Dirac equation, Kemmer-Duffin spin 0 and spin 1 equations, equations for vector-bispinor $\left[{ }^{4,5}\right]$ and the Hurley-type equations $\left[{ }^{6,7}\right]$. In the case of superspins 1 and 0 , the component equations have subsidiary conditions. As it is known $\left[{ }^{8}\right]$, the equations with subsidiary conditions may lead, in the presence of external fields, to various inconsistencies and for that reason the equations without subsidiary conditions are preferred.

It should be mentioned that in the superfield theory the spinor superfield is quite important. In the non-minimal formulation of supergravity, for example, vector and spinor superfields are used as prepotentials [ ${ }^{9}$ ].

The paper is organised as follows: at the beginning we introduce the general spinor superfield and then we proceed with the equations for superspins 1,0 and $1 / 2$, respectively. The notations we use are the same as in $\left[{ }^{1-3}\right]$. 


\section{Superspin 1}

The spinor superfield with an external bispinor index $\alpha$ has a general decomposition

$$
\begin{gathered}
\Psi_{\alpha}(x, \theta)=\psi_{\alpha}^{1}+\bar{\theta} \psi_{\alpha}^{2}+\frac{1}{4} \bar{\theta} \theta \psi_{\alpha}^{3}+\frac{1}{4} \bar{\theta} \gamma^{5} \theta \psi_{\alpha}^{4}+ \\
+\frac{1}{4} \bar{\theta} i \gamma^{v} \gamma^{5} \theta \psi_{v \alpha}+\frac{1}{4} \bar{\theta} \theta \cdot \bar{\theta} \psi_{\alpha}^{6}+\frac{1}{32}(\bar{\theta} \theta)^{2} \psi_{\alpha}^{7},
\end{gathered}
$$

where $\psi_{\alpha}^{1}, \psi_{\alpha}^{3}, \psi_{\alpha}^{4}, \quad \psi_{v \alpha}$ and $\psi_{\alpha}^{7}$ are Fermi fields, and $\psi_{\beta \alpha}^{2}$ and $\psi_{\beta \alpha}^{6}$ are Bose fields.

In the case of superspins 1 and 0 it is useful to represent the Bose fields as follows

where

$$
\psi_{\beta \alpha}^{2}=U_{\alpha \delta} C_{\delta \beta}, \quad \psi_{\beta \alpha}^{6}=V_{\alpha \delta} C_{\delta \beta},
$$

$$
\begin{aligned}
& U=u^{1}+\gamma^{5} u^{2}+\gamma^{\mu} u_{\mu}^{3}+\gamma^{\mu} \gamma^{5} u_{\mu}^{4}+\frac{1}{2} \sigma^{\mu v} u_{\mu v}, \\
& V=v^{1}+\gamma^{5} v^{2}+\gamma^{\mu} v_{\mu}^{3}+\gamma^{\mu} \gamma^{5} v_{\mu}^{4}+\frac{1}{2} \sigma^{\mu v} v_{\mu v} .
\end{aligned}
$$

The spinor superfield is reducible, and it decomposes into the superfields with superspins $1,1 / 2,1 / 2$ and 0 . In this section we deal with the superspin $Y=1$.

The superspin $Y=1$ is singled out by the projection operator $\left[{ }^{1,10}\right]$

$$
E_{1}^{1}=\frac{3}{4}\left(1+\frac{1}{4 \square}(\bar{D} D)^{2}\right)-\frac{1}{8 \square} \partial^{\mu} \sigma_{\mu \nu} \gamma^{5} \bar{D} i \gamma^{v} \gamma^{5} D
$$

In the decomposition (1) the component fields corresponding to $Y=1$ are expressed in the following way -

$$
\begin{gathered}
\underline{\psi}_{\alpha}^{1}=\frac{3}{8}\left(\psi_{\alpha}^{1}+\frac{1}{\square} \psi_{\alpha}^{7}\right)-\frac{1}{4 \square} \partial^{\mu} \sigma_{\mu \nu} \gamma^{5} \psi_{\alpha}^{v}, \\
\underline{U}_{\alpha \beta}=\frac{3}{4}\left(U+\frac{i}{\square} V \hat{\partial}\right)_{\alpha \beta}, \\
\underline{\psi}_{v \alpha}=\frac{3}{4}\left(\eta_{v \sigma}-\frac{\partial_{v} \partial_{\sigma}}{\square}\right) \psi_{\alpha}^{6}-\frac{1}{8} \gamma^{5}\left(\hat{\partial} \gamma^{v}-\partial^{v}\right)\left(\psi_{\alpha}^{1}+\frac{1}{\square} \psi_{\alpha}^{7}\right)+ \\
+\frac{1}{4 \square} \hat{\partial} \gamma^{\mu} \gamma^{5} \varepsilon_{\mu \nu \lambda \sigma} \partial^{\lambda} \psi_{\alpha}^{\sigma} .
\end{gathered}
$$

The other component fields are

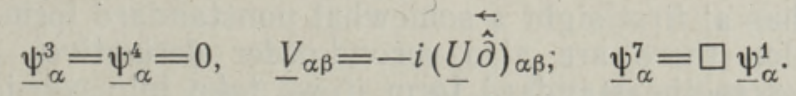

The component fields $\psi_{\alpha}^{1}$ and $\psi_{v \alpha}$ are not independent but satisfy subsidiary conditions derivable from (5)

$$
\gamma^{v} \underline{\psi}_{v}=\hat{\partial} \gamma^{5} \underline{\Psi}^{1}, \quad \partial^{v} \underline{\psi}_{v}=0
$$

(the bispinor index is omitted).

As it is known $\left[{ }^{10}\right]$, the Poincaré spins, corresponding to $Y=1$ are $1 / 2,1,1,3 / 2$. The role of subsidiary conditions (7) is to eliminate two spins $1 / 2$ from $\underline{\psi}_{\alpha}^{1}$ and $\psi_{v \alpha}$. From the transformation properties of component fields it appears that in $U$ the component fields $u^{1}$ and $u^{2}$ are absent since they correspond to the Poincaré spin 0 . 


\section{Equation for superspin 1}

For the beginning, we shall give some remarks on the superfield equations of motion. The root method, given by V. I. Ogievetsky and E. Sokatchev [ $\left.{ }^{1}\right]$, uses the projection operators $E^{Y}$ which singled out different superspins. As the projection operators $E^{Y}$ are nonlocal, the method is applicable when the nonlocal terms are not higher than $\square^{-1}$. Then one can write down the generalized Klein-Gordon equation $\left(\square E^{Y}+m^{2}\right) \Psi=0$ which then may be linearized if $\Psi$ contains at least two superspins $Y$. For that reason this method is directly applicable only in the case of spinor superfield, because in the case of higher superfields the projection operators $E^{Y}$ contain higher nonlocal terms than $\square^{-1}$, if we do not impose at the beginning additional conditions to separate fixed Poincaré spins. Such additional conditions are proposed in $\left[{ }^{10}\right]$.

The general linearized equation for superspin $Y=1$ was derived in [ $\left.{ }^{1}\right]$ : Similarly as in $\left[^{1}\right]$, we choose the parameters $\xi=\eta=0$, and then the equation takes the following form

$$
\frac{1}{8}\left[\left(6 i \hat{\partial}-i \gamma_{\nu} \gamma^{5} \bar{D} i \gamma^{v} \gamma^{5} D\right)+\left(\bar{D} D+3 \gamma^{5} \bar{D} \gamma^{5} D\right)\right]_{\alpha \beta} \Psi_{\beta}=m \Psi_{\alpha} .
$$

Equation (8) is playing a twofold role - it separates superspin 1 and guarantees that the components have mass $m$.

If we write (8) as $\pi \Psi=m \Psi$, we have $\pi^{2}=-\square E_{1}{ }^{1}$ and therefore one can substitute $\Psi \rightarrow \Psi^{1}$, where $\Psi^{1}=E_{1}{ }^{1} \Psi$. In the case of $\Psi^{1} \bar{D} D \Psi^{1}=$ $=\bar{D} \gamma^{5} D \Psi^{1}=0\left[{ }^{10}\right]$ and for $\Psi^{1}$, the equation (8) reduces to

$$
\frac{1}{8}\left(6 i \hat{\partial}-i \gamma_{\nu} \gamma^{5} \bar{D} i \gamma^{v} \gamma^{5} D\right)_{\alpha \beta} \Psi_{\beta}^{1}=m \Psi_{\alpha}^{1} .
$$

Now we write (9) in terms of component fields $\psi_{\alpha}^{1}, \psi_{v \alpha}$ and $U_{\alpha \beta}$. In the following part of this section, we operate only with the component fields corresponding to $Y=1$ and for that reason we denote them simply $\psi_{\alpha}$, $\psi_{v \alpha}$ and $U_{\alpha \beta}$

$$
\begin{aligned}
& \frac{3 i}{4} \hat{\partial} \psi^{1}-\frac{i}{4} \gamma^{v} \gamma^{5} \psi_{\nu}=m \psi^{1}, \\
& \frac{3 i}{4} \hat{\partial} \psi^{\mu}-\frac{i}{4}\left(\gamma^{\mu} \gamma^{5} \square-\hat{\partial} \gamma^{5} \partial^{\mu}\right) \psi^{1}+\frac{i}{4} \gamma \nu \gamma^{5} \varepsilon^{v \mu \lambda \rho} \partial_{\lambda} \psi_{\rho}=m \psi^{\mu}, \\
& \frac{3 i}{4} \hat{\partial} U+\frac{i}{4} \gamma_{\nu} \gamma^{5} U \gamma^{5} \gamma^{\nu} \hat{\hat{\partial}}-\frac{i}{4} \hat{\partial} \gamma^{5} U \gamma^{5}=m U .
\end{aligned}
$$

From (10) one can see that the system we have obtained by the direct substitution, has at first sight a somewhat nonstandard form, since in the second equation there are also second-order derivatives present. The system reduces to the standard form if we take into consideration that the fields $\psi_{\alpha}{ }^{1}$ and $\psi_{v \alpha}$ are not independent; but satisfy subsidiary conditions (7)

$$
\gamma^{v} \psi_{v}=\hat{\partial} \gamma^{5} \psi^{1}, \quad \partial^{v} \psi_{v}=0 .
$$

At first we deal with the Fermi fields $\psi_{\alpha}{ }^{1}$ and $\psi_{v \alpha}$. If we use the first subsidiary condition (11), we obtain from (10)

$$
\begin{aligned}
& (i \hat{\partial}-m) \psi^{1}=0, \\
& (i \hat{\partial}-m) \psi^{\mu}-\frac{i}{4} \psi^{\mu} \partial^{v} \psi_{v}=0 .
\end{aligned}
$$


The component field $\psi^{1}$ satisfies the Dirac equation and describes spin $1 / 2$ with mass $m$. The equation obtained for $\psi^{\mu}$, describes the following states: spin $3 / 2$ state with mass $m$, and two spin $1 / 2$ states with masses $m$ and $4 m / 3$. It is easy to verify that the second subsidiary condition (11) eliminates the spin $1 / 2$ state with mass $4 \mathrm{~m} / 3$. Indeed, if we apply $\partial_{\mu}$ to the second equation (12), we obtain $(i \hat{\partial}-4 m / 3) \partial_{\mu} \psi^{\mu}=0$. Consequently, $\partial_{\mu} \psi^{\mu}$ corresponds to the mass $4 \mathrm{~m} / 3$ and is eliminated due to $\partial_{\mu} \psi^{\mu}=0$. The remaining spin $1 / 2$ state in $\psi_{v}$ is not independent but related to $\psi^{1}$, and therefore the system (12) with șubsidiary conditions (11) describes two spins: $1 / 2$ and $3 / 2$ with the same mass $m$.

The equations (12) and (11) may be also written in equivalent but simpler form -

$$
\begin{array}{ll}
(i \hat{\partial}-m) \psi^{1}=0, & (i \hat{\partial}-m) \psi^{\mu}=0, \\
\gamma^{v} \psi_{v}=\hat{\partial} \gamma^{5} \psi^{1}, & \partial^{v} \psi_{v}=0 .
\end{array}
$$

Now we shall deal with the equations for the Bose fields. For $Y=1$ $u^{1}=u^{2}=0$ and $U$ has a decomposition

$$
U=\gamma^{\mu} u_{\mu}^{3}+\gamma^{\mu} \gamma^{5} u_{\mu}^{4}+\frac{1}{2} \sigma^{\mu v} u_{\mu v}
$$

The third equation (10) gives the following component equations

$$
\begin{aligned}
& i \partial^{\mu} u_{\mu v}=m u_{v}^{3}, \\
& -\frac{i}{2} \partial_{\mu} \varepsilon^{\mu v \rho \sigma} u_{\rho \sigma}=m u^{4 v}, \\
& i\left(\partial^{\mu} u^{3 v}-\partial^{v} u^{3 \mu}\right)+i \varepsilon^{\mu v \rho \sigma} \partial_{\rho} u_{\sigma}^{4}=m u^{\mu v} .
\end{aligned}
$$

The system (15) decomposes into the two independent spin 1 equations corresponding to the representations $(1,0) \oplus(1 / 2,1 / 2)$ and $(0,1) \oplus$ $\oplus(1 / 2,1 / 2)$. The equations obtained are the Hurley-type equations $\left[{ }^{6,7}\right]$.

In order to decompose the system (15), we define the new quantities, dual tensor

$$
\tilde{u}^{\mu \nu}=\frac{1}{2} \varepsilon^{\mu v \rho \sigma} u_{\rho \sigma}
$$

tensors

$$
a^{\mu v}=\frac{1}{\sqrt{2}}\left(u^{\mu v}-i \widetilde{u}^{\mu v}\right), \quad b^{\mu v}=\frac{1}{\sqrt{2}}\left(u^{\mu v}+i \widetilde{u}^{\mu v}\right),
$$

and vectors

$$
a^{\mu}=\frac{1}{\sqrt{2}}\left(u^{3 \mu}+i u^{4 \mu}\right), \quad b^{\mu}=\frac{1}{\sqrt{2}}\left(u^{3 \mu}-i u^{4 \mu}\right) .
$$

The tensors $a^{\mu v}$ and $b^{\mu v}$ transform according to the representations $(0,1)$ and $(1,0)$, respectively.

Now the equations take the form

$$
\begin{aligned}
& i \partial^{\mu} a_{\mu \nu}=m a_{v}, \\
& i\left(\partial^{\mu} a^{v}-\partial^{v} a^{\mu}\right)+\varepsilon^{\mu v \rho \sigma} \partial_{\rho} a_{\sigma}=m a^{\mu v}, \\
& i \partial^{\mu} b_{\mu v}=m b_{v}, \\
& i\left(\partial^{\mu} b^{v}-\partial^{v} b^{\mu}\right)-\varepsilon^{\mu v \rho \sigma} \partial_{\rho} b_{\sigma}=m b^{\mu v} .
\end{aligned}
$$


As we can see, the equations (15) reduce into two independent equations indeed. It is easy to verify that they describe two independent spin 1 states with the same mass $m$, and are equivalent to the doubled Hurleytype equations.

In conclusion: for $Y=1$ we have obtained the set of equations which described four states with the mass $m$ and Poincaré spins $1 / 2,1,1$, and 3/2.

As we have mentioned in introduction, the same equation has been derived and analysed in [ $\left.{ }^{1}\right]$. By the proper redefinition of component fields, it is demonstrated that the Fermi fields satisfy the Dirac and Rarita-Schwinger equations, and the Bose fields satisfy the Proca equation. Since the redefinition of fields seems somewhat artifical, we investigated the equations for component fields which directly followed from the linearized superfield equation (8). As we have seen, the component equations are reduced to the equations which have been previously investigated in the theory of invariant wave equations. The only defect of our equations is that they are equations with subsidiary conditions.

\section{Superspin 0}

The superspin 0 case is treated similarly as the superspin 1 case. $Y=0$ is singled out by the projection operator $\left[2,{ }^{20}\right]$

$$
E_{1}^{0}=\frac{1}{4}\left(1+\frac{1}{4 \square}(\bar{D} D)^{2}\right)+\frac{1}{8 \square} \partial^{\mu} \sigma_{\mu \nu} \gamma^{5} \bar{D} i \gamma^{v} \gamma^{5} D
$$

The component fields corresponding to $Y=0$ are expressed in the following way (we also denote them $\underline{\psi}$ )

$$
\begin{aligned}
& \underline{\psi}_{\alpha}^{1}=\frac{1}{8}\left(\psi_{\alpha}^{1}+\frac{1}{\square} \psi_{\alpha}^{7}\right)+\frac{1}{4 \square} \partial^{\mu} \sigma_{\mu \nu} \gamma^{5} \psi_{\alpha}^{v}, \\
& \underline{U}_{\alpha \beta}=-\frac{1}{4}\left(U+\frac{i}{\square} V \hat{\partial}\right)_{\alpha \beta}, \\
& \underline{\psi}_{v \alpha}=\frac{1}{8}\left(\eta_{v \sigma}-\frac{\partial_{v} \partial_{\sigma}}{\square}\right)_{\alpha}^{\sigma}+\frac{1}{8} \gamma^{5}\left(\hat{\partial} \gamma^{v}-\partial^{v}\right)\left(\psi_{\alpha}^{1}+\frac{1}{\square} \psi_{\alpha}^{7}\right)- \\
& -\frac{1}{4 \square} \hat{\partial} \gamma^{\mu} \gamma^{5} \varepsilon_{\mu \nu \rho \sigma} \partial^{\rho} \psi_{\alpha}^{\sigma} .
\end{aligned}
$$

The other component fields are

$$
\underline{\psi}_{\alpha}^{3}=\underline{\psi}_{\alpha}^{4}=0, \quad \underline{V}_{\alpha \beta}=-i(\underline{U \hat{\partial}})_{\alpha \beta}, \quad \underline{\psi}_{\alpha}^{7}=\square \underline{\psi}_{\alpha}^{1} .
$$

Now the component fields $\underline{\psi}_{\alpha}^{1}$ and $\underline{\psi}_{v \alpha}$ satisfy the following subsidiary conditions derivable from (19) -

$$
\gamma^{v} \underline{\psi}_{v}=3 \gamma^{5} \underline{\partial}_{\underline{\psi}}, \quad \partial^{v} \underline{\psi}_{v}=0 .
$$

The Poincaré spins corresponding to $Y=0$ are 0,0 and $1 / 2$. Subsidiary conditions (21) connect spins $1 / 2$ in $\underline{\psi}_{\alpha}^{1}$ and $\psi_{v \alpha}$. From the transformation properties of component fields it follows that in $U$ the component field $u_{\mu \nu}$ is absent since it corresponds to the Poincaré spin 1. 


\section{Equation for superspin 0}

The general linearized equation for superspin $Y=0$ was derived in $\left[{ }^{2}\right]$. Similarly as in the $Y=1$ case, we choose the parameters $\xi=\eta=0$ and the superfield equation takes the following form -

$$
\frac{1}{8}\left[\left(2 i \hat{\partial}+i \gamma_{v} \gamma^{5} \bar{D} i \gamma^{v} \gamma^{5} D\right)+\left(\bar{D} D-\gamma^{5} \bar{D} \gamma^{5} D\right)\right]_{\alpha \beta} \Psi_{\beta}=m \Psi_{\alpha}
$$

Since the equation (22) describes superspin 0 , one can substitute $\Psi \rightarrow \Psi^{0}$, where $\Psi^{0}=E_{1}{ }^{0} \Psi$. In the case of $Y=0, \Psi^{0}$ satisfies $\bar{D} D \Psi^{0}=$ $=\bar{D} \gamma^{5} D \Psi^{0}=0$, and for $\Psi^{0}$ the equation (22) reduces to

$$
\frac{1}{8}\left(2 i \hat{\partial}+i \gamma v \gamma^{5} \bar{D} i \gamma^{v} \gamma^{5} D\right)_{\alpha \beta} \Psi_{\beta}^{0}=m \Psi_{\alpha}^{0}
$$

Now we write $(23)$ in terms of component fields $\underline{\psi}_{\alpha}^{1}, \underline{\psi}_{v \alpha}$ and $\underline{U}_{\alpha \beta}$ which we denote in this section simply as $\psi_{\alpha}^{1}, \psi_{v \alpha}$ and $\bar{U}$,

$$
\begin{aligned}
& \frac{i}{4} \hat{\partial} \psi^{1}+\frac{i}{4} \gamma^{v} \gamma^{5} \psi_{v}=m \psi^{1}, \\
& \frac{i}{4} \hat{\partial} \psi^{\mu}+\frac{i}{4}\left(\gamma^{\mu} \gamma^{5} \square-\hat{\partial} \gamma^{5} \partial^{\mu}\right) \psi^{1}-\frac{i}{4} \gamma v \gamma^{5} \varepsilon^{v \mu \rho \sigma} \partial_{\rho} \psi_{\sigma}=m \psi^{\mu} \\
& \frac{i}{4} \hat{\partial} U+\frac{i}{4} \hat{\partial} \gamma^{5} U \gamma^{5}-\frac{i}{4} \gamma_{v} \gamma^{5} U \gamma^{5} \gamma^{v} \hat{\hat{\partial}}=m U
\end{aligned}
$$

To the given system one must add the following subsidiary conditions

$$
\gamma^{v} \psi_{v}=3 \gamma^{5} \hat{\partial} \psi^{1}, \quad \partial^{v} \psi_{v}=0 .
$$

At first we shall consider the Fermi fields $\psi_{\alpha}{ }^{1}$ and $\psi_{v \alpha}$. If we use the first subsidiary condition (25), we obtain from (24)

$$
\begin{aligned}
& (i \hat{\partial}-m) \psi^{1}=0, \\
& \frac{i}{3}\left(\partial^{\mu}-\gamma^{\mu} \hat{\partial}\right) \gamma^{v} \psi_{v}+\frac{i}{4} \gamma^{\mu} \partial^{v} \psi_{v}=m \psi^{\mu} .
\end{aligned}
$$

For $\psi^{1}$ we have again the Dirac equation. The equation for vector-bispinor $\psi_{v}$ is interesting, since it describes only spin $1 / 2$ states (as it is known in the $Y=0$ case, spin $3 / 2$ is absent). The second equation (26) describes two spin $1 / 2$ states with masses $m$ and $4 m$. It is possible to verify that the second subsidiary condition (25) eliminates the state with mass $4 \mathrm{~m}$. Indeed, if we apply $\partial_{\mu}$, we obtain $(i \hat{\partial}-4 m) \partial_{\mu} \psi^{\mu}=0 . \partial_{\mu} \psi^{\mu}$ describes mass $4 m$ and is eliminated due to $\partial_{\mu} \psi^{\mu}=0$. Since the remaining spin states of $\psi^{1}$ and $\psi_{v}$ are related, only one spin $1 / 2$ state with mass $m$ remains. Therefore, only the component field is necessary and one may leave in (26) only the Dirac equation for $\psi^{1}$.

The equations (26) with subsidiary conditions (25) may be written also in the following equivalent form -

$$
\begin{aligned}
& (i \hat{\partial}-m) \psi^{1}=0, \\
& \frac{i}{3}\left(\partial^{\mu}-\gamma^{\mu} \hat{\partial}\right) \psi^{v} \psi_{v}=m \psi^{\mu}, \\
& \gamma^{v} \psi_{v}=3 \gamma^{5} \hat{\partial} \psi^{1}, \quad \partial^{v} \psi_{v}=0 .
\end{aligned}
$$


It is interesting to note that now the equation for $\psi_{v}$ describes only one spin $1 / 2$ state with mass $m$, and is equivalent to the barnacled new spin $1 / 2$ equation proposed previously by A. Z. Capri $\left[{ }^{7,11}\right]$. The Capri equation in turn is equivalent to the Dirac equation $\left.{ }^{7}\right]$.

For Bose fields $U$ is decomposed as

$$
U=u^{1}+\gamma^{5} u^{2}+\gamma^{\mu} u_{\mu}^{3}+\gamma^{\mu} \gamma^{5} u_{\mu}^{4},
$$

and the third equation (24) gives us two Kemmer-Duffin spin 0 equations

$$
\begin{array}{ll}
i \partial^{\mu} u_{\mu}^{3}=m u^{1} & i \partial^{\mu} u_{\mu}^{4}=m u^{2} \\
i \partial_{\mu} u^{1}=m u_{\mu}^{3} & i \partial_{\mu} u^{2}=m u_{\mu}^{4} .
\end{array}
$$

In conclusion: for $Y=0$ we have obtained the set of equations which described three states with the same mass $m$ and Poincaré spins 0,0 and $1 / 2$.

In the case of $Y=1$ we have obtained for Bose fields the doubled Hurley-type equation. It should be mentioned that the Kemmer-Duffin spin 0 equations obtained for superspin $Y=0$ are in principle of the same type $\left[{ }^{7}\right]$.

\section{Superspin $1 / 2$}

In the case of superspin $1 / 2$, we have two independent superfields - the chiral superfields $\Psi_{+}$and $\Psi_{-}$. They are projected out with the help of the following projection operators $\left[{ }^{3,10}\right]$ -

$$
E_{ \pm}=-\frac{1}{8 \square}(\bar{D} D)^{2} \mp \frac{i}{4 \square} \partial_{\mu} \bar{D} i \gamma^{\mu} \gamma^{5} D
$$

Now the independent component fields are expressed as

$$
\begin{aligned}
& \psi_{ \pm \alpha}^{1}=\frac{1}{4}\left(\psi_{\alpha}^{1}-\frac{1}{\square} \psi_{\alpha}^{7}\right) \mp \frac{1}{2 \square} \partial^{v} \psi_{v \alpha}, \\
& \psi_{ \pm \beta \alpha}^{2}=\frac{1}{2}\left[\frac{1 \pm i \gamma^{5}}{2}\left(\psi^{2}-\frac{1}{i \hat{\partial}} \psi^{6}\right)\right]_{\beta \alpha} \\
& \psi_{ \pm \alpha}^{3}=\frac{1}{2}\left(\psi_{\alpha}^{3} \mp i \psi_{\alpha}^{4}\right)
\end{aligned}
$$

The other component fields are dependent

$$
\begin{array}{ll}
\psi_{ \pm \alpha}^{4}= \pm i \psi_{ \pm \alpha}^{3}, & \psi_{ \pm v \alpha}= \pm i \partial_{\nu} \psi_{ \pm \alpha}^{1}, \\
\psi_{ \pm \beta \alpha}^{6}=-i\left(\hat{\partial} \psi_{ \pm}^{2}\right)_{\beta \alpha}, & \psi_{ \pm \alpha}^{7}=-\square \psi_{ \pm \alpha}^{1} .
\end{array}
$$

In the following we deal with the equation proposed by A. Salam and J. Strathdee $\left[{ }^{3,12}\right]$, and in that case the redefinition of component field $\psi^{2}$ is not necessary.

\section{Equation for superspin $1 / 2$}

The linearized equation derived with the help of root method $\left[{ }^{2}\right]$ is not physically interesting, because the equations for component fields do not link the chiral components $\psi_{+}$and $\psi_{-}$. For that reason we deal with the Salam-Strathdee equation for chiral superfields $\Psi_{+}$and $\Psi_{-}\left[{ }^{3,12}\right]-$ 


$$
\begin{aligned}
& (i \hat{\partial}-2 m) \Psi_{+}+\frac{1}{2} \bar{D} D \Psi_{-}=0 \\
& (i \hat{\partial}-2 m) \Psi_{-}+\frac{1}{2} \bar{D} D \Psi_{+}=0
\end{aligned}
$$

In terms of component fields we obtain

$$
\begin{aligned}
& (i \hat{\partial}-2 m) \psi_{ \pm}^{1}-\psi_{\mp}^{3}=0, \\
& (i \hat{\partial}-2 m) \psi_{ \pm}^{3}+\square \psi_{\mp}^{1}=0, \\
& (i \hat{\partial}-2 m)_{\alpha \gamma} \psi_{ \pm \beta \gamma}^{2}+i \hat{\partial}_{\beta \gamma} \psi_{ \pm \nu \alpha}^{2}=0
\end{aligned}
$$

The structure of equations (34) is in some sense analogous to the Wess-Zumino equations for chiral scalar superfield. In the case of Fermi fields, the equations (34) may be rewritten as

$$
(i \hat{\partial}-m) \psi_{ \pm}^{1}=0, \quad m \psi_{ \pm}^{1}=-\psi_{\mp}^{3} .
$$

$\psi_{ \pm}^{1}$ satisfy the Dirac equation, the component fields $\psi_{ \pm}^{3}$ are dependent. Therefore we have two spin $1 / 2$ states with the same mass $m$.

In the case of Bose fields, the chiral components $\psi_{+\beta \alpha}^{2}$ and $\psi_{-\beta \alpha}^{2}$ formed a second rank bispinor

$$
\psi_{\beta \alpha}=\psi_{+\beta \alpha}^{2}+\psi_{-\beta \alpha}^{2}
$$

which from (34) satisfies the following equation -

$$
i \hat{\partial}_{\alpha \gamma \psi_{\beta \gamma}}+i \hat{\partial}_{\beta \gamma} \psi_{\nu \alpha}=2 m \psi_{\beta \alpha} .
$$

The last equation is the SO $(1,4)$-type equation which describes spins 0 and 1 , and is equivalent to the Kemmer-Duffin spin 0 and spin 1 equations [13]. The SO $(1,4)$ structure of equation $(37)$ may be easily demonstrated if we write (37) in the form

where the matrices $\beta^{\mu}$ are

$$
i \partial_{\mu} \beta^{\mu} \psi=m \psi,
$$

$$
\beta^{\mu}=\frac{1}{2}\left(\gamma^{\mu} \times I+I \times \gamma^{\mu}\right)
$$

Using the properties of $\gamma$ matrices, it is easy to verify that the generators $S^{\mu 5}=\beta^{\mu}$ generate the $\mathrm{SO}(1,4)$ algebra, since they give $\left[S^{\mu 5}, S^{\nu 5}\right]=$ $=S^{\mu \nu}$, where $S^{\mu \nu}$ are the generators of the Lorentz group. The equivalence of (37) with the Kemmer-Duffin equations has also been stated in $\left[{ }^{14}\right]$.

In conclusion: $Y=1 / 2$ equation for chiral superfields $\Psi_{+}$and $\Psi_{-}$ describes four states with the mass $m$ and Poincaré spins $0,1 / 2,1 / 2$, and 1 .

\section{Conclusions}

In this paper we have investigated the equations for component fields which followed from the spinor superfield equations of motion. The equations of Fermi fields have been reduced to the Dirac equation and multi-mass equations for vector-baspinor. The equations of Bose fields were: Hurley-type equations in the superspin 1 case, Kemmer-Duffin spin 0 equations in the superspin 0 case, and Kemmer-Duffin spin 0 and spin 1 equations in the superspin $1 / 2$ case. 
The general structure of component equations was different. The equations for $Y=0$ and $Y=1$ were equations with subsidiary conditions, the equations for chiral superfield $(Y=1 / 2)$ had no subsidiary conditions. In the theory of invariant wave equations it is known that in the presence of external fields the equations with subsidiary conditions may lead to algebraic inconsistencies, and for that reason the equations without subsidiary conditions were preferred. We are of opinion that the consistency problem of superfield equations when an external field is present is quite important and needs further investigation.

\section{REFERENCES}

1. Ogievetsky, V. I., Sokatchev, E. J. Phys. A : Math. Gen., 10, № 11, 2021-2030 (1977).

2. Loide, R.-K., Suurvarik. P. ENSV TA Toim. Füüs. Matem., 32, № 2, 165-171 (1983).

3. Salam, A., Strathdee, J. Phys. Rev., D11, № 6, $1521-1535$ (1975).

4. Kõiv, M., Loide, R.-K., Saar, R. ENSV TA Toim. Füüs. Matem., 31, № 3, 300-303 (1982).

5. Kõiv, M., Loide, R.-K., Saar, R. Preprint F-17, Tartu, 1982.

6. Hurley, W. J. Phys. Rev., D4, № 12, 3605-3616 (1971).

7. Loide, K., Loide, R.-K. Preprint F-6, Tartu, 1977.

8. Wightman, A. S. Invariant Wave Equations; General Theory and Applications to the External Field Problem. Lecture Notes in Physics, vol. 73. Berlin, Heidelberg, New York, 1978.

9. Siegel, W., Gates, J. Nucl. Phys., B147, № 1, 77-104 (1979).

10. Sokatchev. E. Nucl. Phys., B99, № 1, 96-108 (1975).

11. Capri, A. Z. Phys. Rev., 187, № 5, 1811-1815 (1969).

12. Salam, A., Strathdee, J. Fortschr. Phys., 26, № 2, 57-142 (1978).

13. Кыйя М.. Лойде Р.-К., Мейтре И. Тр. Таллин. политех. ин-та, сер. А., № 289, $11-27$ (1970).

14. Adjei, S. A., Akyeampong, D. A. Nuovo cim., 26A, № 1, 84-93 (1975).

Tallinn Technical University

Academy of Sciences of the Estonian SSR,

Received Institute of Physics

\section{R.-K. LOIDE, P. SUURVARIK}

\section{SPIINORSUPERVÄLJA LIIKUMISVORRANDITEST}

Töös on analüüsitud spiinorsupervälja liikumisvōrrandite struktuuri komponentväljade kaudu, mille vōrrandid taanduvad invariantsete lainevōrrandite teoorias varem uuritud võrranditele. Superspinnide 1 ja 0 korral saame Diraci võrrandi, mitme osakese vôrrandi vektor-bispiinorile ja Hurley vōrrandi tüüpi võrrandid. Superspinni $1 / 2 \mathrm{korral}$ aga saame Diraci võrrandi ning Kemmer-Duffini spinn̊ 0 ja spinni 1 võrrandi.

\section{Р.-К. ЛОИДЕ, П. СУУРВАРНК}

\section{ОБ УРАВНЕНИЯХ ДВИЖЕНИЯ ДЛЯ СПИНОРНОГО СУПЕРПОЛЯ}

Рассмотрена структура уравнений движения спинорного суперполя с помощьњ компонентных полей, уравнения которых приводятся к уравнениям, ранее исследованным в теории инвариантных волновых уравнений. Для суперспинов 1 и 0 получены уравнение Дирака. многочастичное уравнение для вектор-биспннора и уравнения типа уравнений Хэрли. Для суперспина 1/2 получены уравнение Дирака и уравнения Кеммера-Дэффина для спинов 0 и 1 . 